\title{
From optical to chemical hot spots in plasmonics
}

\author{
Julian Gargiulo, Rodrigo Berté, Yi Li, Stefan A. Maier, Emiliano Cortés* \\ Chair in Hybrid Nanosystems, Nanoinstitute Munich, Faculty of Physics, Ludwig-Maximilians-Universität \\ München, 80539 München, Germany
}

\section{Conspectus}

In recent years, the possibility to induce chemical transformations by using tunable plasmonic modes has opened the question of whether we can control or create chemical hot spots in these systems. This can be rationalized as the reactive analogue of the well-established concept of optical hot spots, which have drawn a great deal of attention to plasmonic nanostructures for their ability to circumvent the far-field diffraction limit of conventional optical elements.

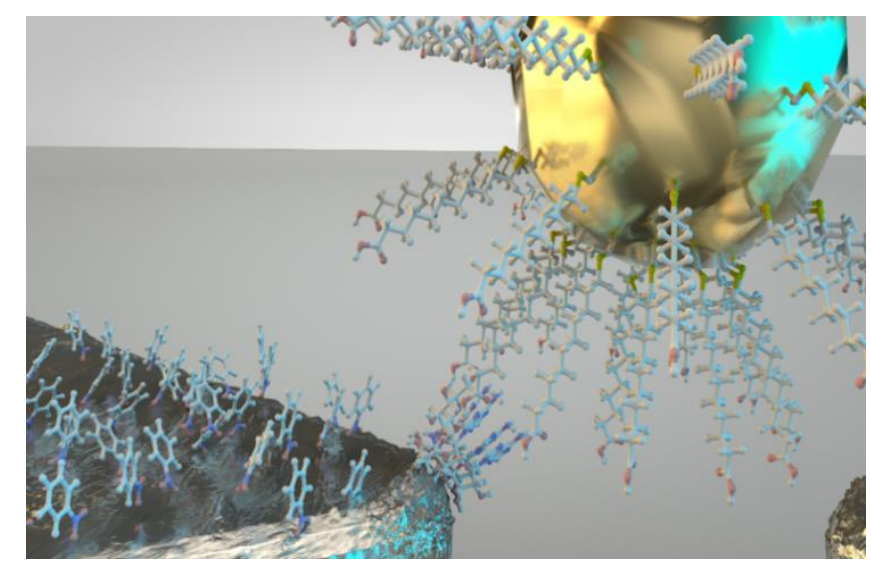

The parameters that determine the reactivity of plasmonic systems are deeply influenced by the dynamics and interplay of photons, plasmon-polaritons, carriers, phonons and molecular states. Although optical hot spots can be mainly defined by the geometry and permittivity of the nanostructures, the degrees of freedom influencing their photocatalytic properties appear to be much more numerous. These degrees of freedom can affect the reaction rates, the product selectivity or the spatial localization of a chemical reaction. In this Account, we show how the control of chemical hot spots can be achieved by carefully tuning the parameters that influence the cascade of events following the optical excitation of plasmonic modes in nanostructures.

We discuss here a series of single photocatalyst techniques and ideas of how plasmonic nanoscale reactivity can be spatially mapped and imaged, and how the lifetime of hot and thermalized carriers can be altered by trap states on semiconductors and by metal-semiconductor interfaces. In addition, the tailored generation of non-thermal phonons in metallic nanostructures and their dissipation is shown as a promise to understand and exploit thermal photocatalysis at the nanoscale. Surpassing or enhancing each of these energy channels should enable to engineer solar nanometric photocatalysts.

Nevertheless, the ultimate capability of a plasmonic photocatalyst to trigger a chemical reaction is correlated to its ability to navigate through, or even modify, the potential energy surface of a given chemical reaction. Here we reunite both worlds, the plasmonic photocatalysts and the molecular ones, identifying different energy transfer pathways and their influence on selectivity and efficiency of chemical reactions. We foresee that the migration from optical to chemical hot spots will greatly assist the understanding of ongoing plasmonic chemistry.

\section{Introduction}

Plasmon assisted catalysis is the field that aims to exploit the properties of localized surface plasmon resonances (LSPRs) for chemical reactions. LSPRs are the light-induced coherent motion of electrons that produce large scattering and absorption cross-sections as well as dramatic enhancements of the electromagnetic field in regions close to the surface of metallic nanostructures. The energy of far-field light can be compressed into sub-wavelength scale via storing part of it as kinetic energy of the free electrons. As such, LSPRs have mixed electromagnetic and free carrier nature. Squeezing of visible light using LSPRs leads to 
extreme localization of the electric field $(|E|)$ on the nanoscale, transforming them into efficient optical nano-antennas. Optical hot spots are usually characterized by the field enhancement $f_{E}=|E| /\left|E_{0}\right|$, being $\left|E_{0}\right|$ the amplitude of the incident electric field. The frequency and spatial distribution of optical hot spots can be tuned by changing the size, shape, material and structure of the nanoparticle (NP) as well as the refractive index of the medium. The small mode volume of LSPRs also increases the photonic local density of states (LDOS). As such, optical hot spots have been instrumental for enhancing or modifying the properties of molecules that depend on the intensity of the electric field and/or it is nanoscale confinement. Enhanced Raman scattering (SERS) and fluorescence emission (SEF) of molecules next to plasmonic NPs are two of the most remarkable examples. ${ }^{1,2}$

During the previous decades, nanofocusing into optical hot spots has been one of the driving forces behind the tremendous advances in nanoscale fabrication methods as well as in the design and discovering of new materials for nano-optics. Recently, the perspective of employing the energy absorbed by LSPRs and guide it towards a nearby molecule to produce a chemical transformation has emerged. ${ }^{3-7}$ Taking profit of the established concept of optical hot spots in plasmonic nano-antennas, we try here to make a link and discuss the role of plasmonic nanostructures as nano-reactors confining energy into chemical hot spots.

\section{Chemical Hot Spots}

Moving from optical to chemical hot spots in plasmonics implies a completely new optimization problem. ${ }^{8}$ While optical hot spots depend only on the properties of the antenna and the incident light, the reactivity of a plasmonic antenna is strongly reaction-dependent (i.e. the reaction pathway is set by the molecules, the antenna and the light). Figure 1 schematically depicts several phenomena that may affect plasmonic reactivity on the nanoscale, each one presenting a distinctive spatial distribution. These include plasmonderived phenomena such as electromagnetic field enhancement, temperature, absorption (hot-electron generation), and mechanical vibrations (phonons). Furthermore, it is a wellknown fact in heterogeneous catalysis that metal NPs in the dark (no illumination) show subparticle inhomogeneous chemical activity given by surface facets and defect-sites, such as low coordinated-atoms. ${ }^{9}$ To illustrate this point, the inset catalytic activity in dark of Figure 1 reproduces the activity map measured by Chen et.al. of a fluorogenic reduction on Au nanotriangles. ${ }^{9}$ The convolution of all the phenomena shown in Figure 1 build up the plasmonassisted chemical map. However, further mechanistic details of the molecules and the plasmonic photocatalysts are needed in order to control and predict reactivity. In the following sections, we will first describe a (photo)chemical reaction from the molecule perspective. Then, we describe how processes triggered after plasmon decay can contribute to catalyze such a reaction. 


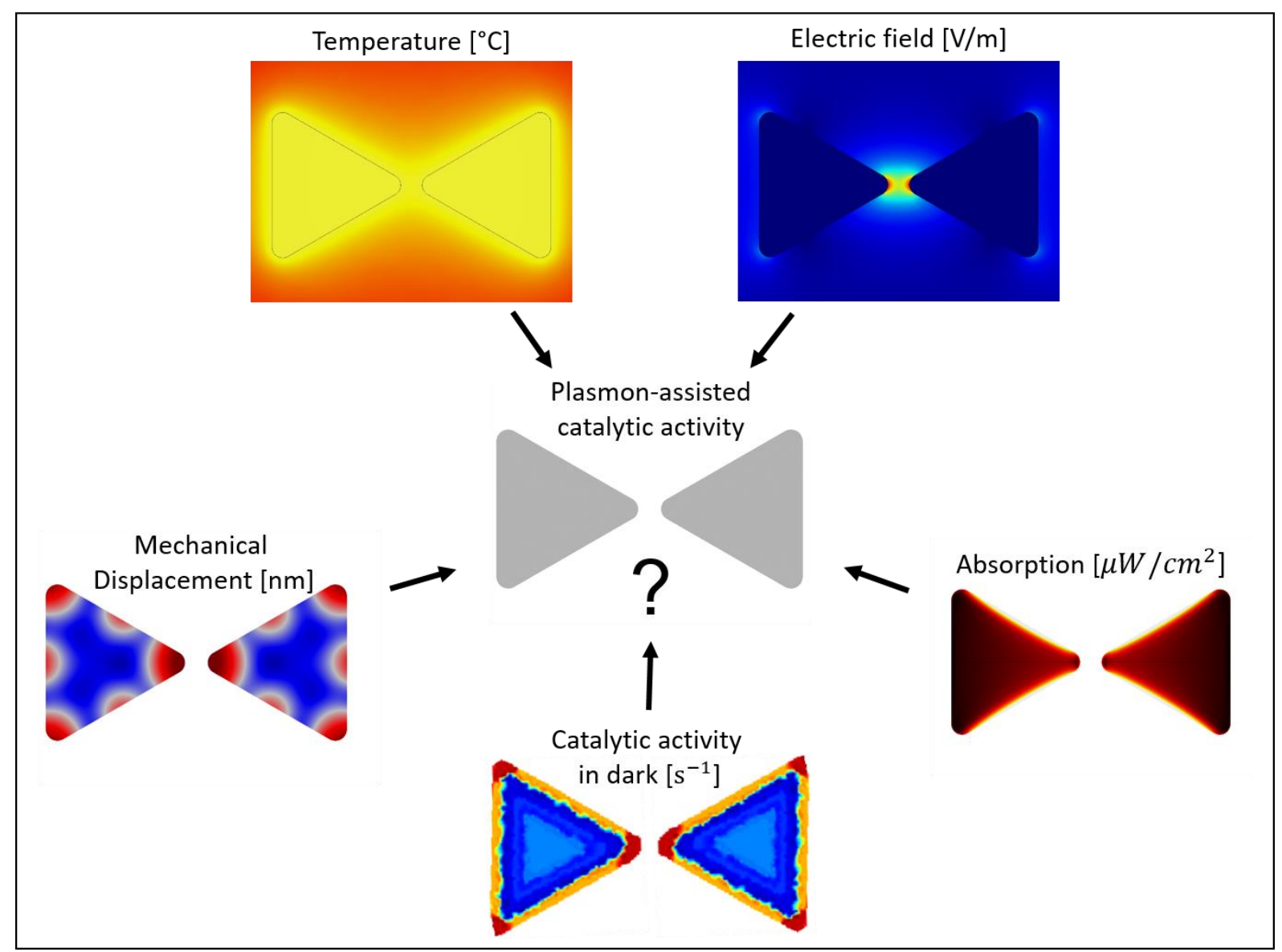

Figure 1: Spatial distribution of different phenomena influencing chemical activity in plasmon-assisted chemistry. Inset "Catalytic activity in dark" adapted with permission from American Chemical Society. ${ }^{10}$

\section{Navigating the potential energy surface}

The mechanism and energetic aspects of a chemical reaction can be described in terms of the potential energy surface (PES) which describes the potential energy of the system as a function of reaction coordinates, as schematized in Figure 2. Temperature or pressure have served for a long time as a vehicle to travel along the PES from an initial state $A$ to a local or global minimum B (i.e. the formation of products). Crossing the highest hill or activation barrier of the PES is usually referred as the rate-limiting step (RLS) of the overall journey, thus being responsible for governing the kinetics of the reaction.

In heterogeneous catalysis, the presence of a catalyst alters the PES by reducing the activation barriers - in particular the RLS one -therefore speeding up the reaction rate and enhancing efficiency. This requires adsorption of the reactant on the catalyst surface, an input of energy to overcome the activation barrier and desorption of the product, releasing the catalytic site for a new cycle to start. Pressure (or reactants concentration), temperature and reaction conditions can influence the adsorption-desorption dynamics (i.e. coverage), thus impacting in the reaction evolution. ${ }^{11}$

Another way of modifying the reaction pathway in heterogeneous catalysis would be accessing to excited electronic states of the reactant-catalyst system, where a new potential energy surface can be explored. ${ }^{12,13}$ This would require an electron (or hole) transfer process in order to form a transient ionic species $\left(A^{*}\right)$. Note that here an electron (hole) is added to 
(removed from) the adsorbed molecule and as such the new PES corresponds to a charged state. This short-living state can then undergo a chemical reaction along its new potential energy surface leading to desorption, dissociation, or even redox reactions (in this last case the charge is permanently transferred and a counter reaction must take place). Interestingly, the activation barriers of the electronically excited PES could be different from the ones in the ground PES. This is shown in Figure 2, where the system is now going to a different minima C. Lowering the activation barrier of the RLS is linked to the efficiency and the overall energy consumption of the reaction, while the appearance of new minima states could lead to selectivity or the formation of new products for the same molecule-catalyst system.

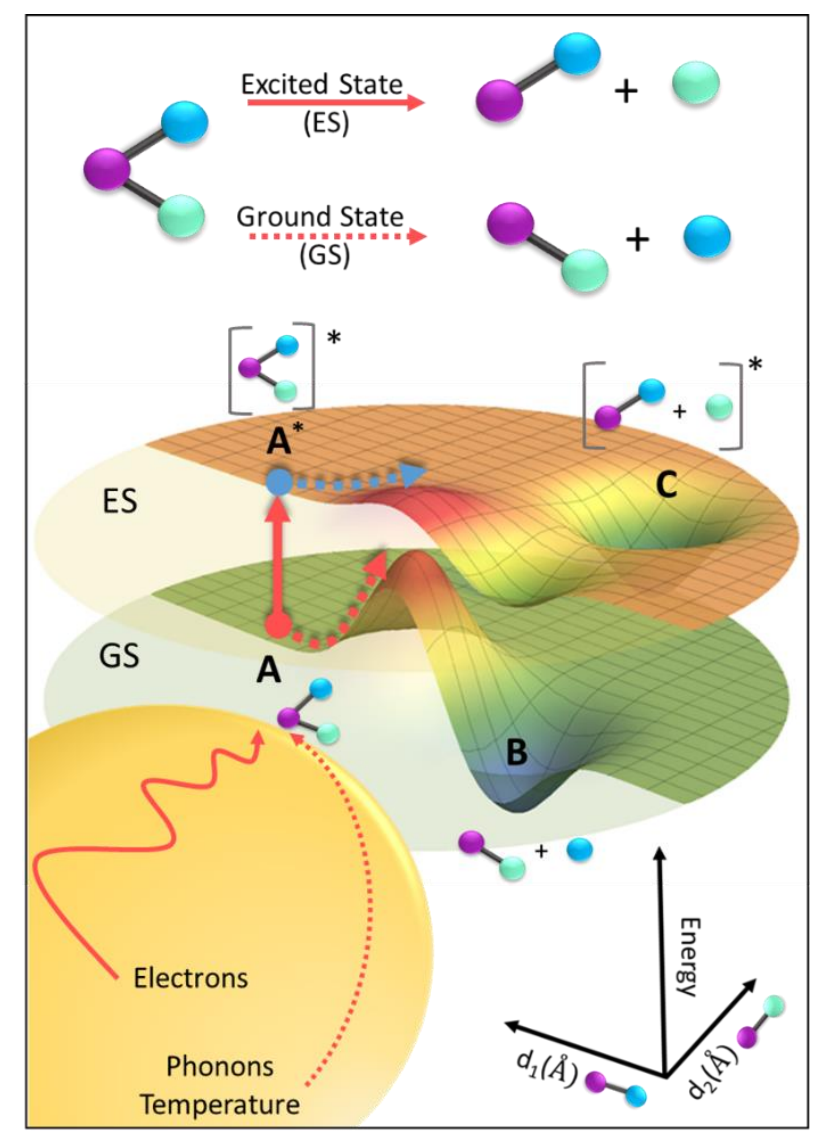

Figure 2: Left: Ground (green, GS) and excited (orange, ES) potential energy surfaces of a chemical reaction (i.e. the dissociation of a triatomic molecule). The excited electronic state may possess a lower activation barrier for the generation of products (linked to efficiency) as well as new valleys (linked to selectivity). Cascade of processes triggered by plasmon decay: electrons, phonons and temperature can be transferred to the molecule opening new pathways to manipulate chemical reactivity with light.

Access to the excited electronic states of the metal-molecule system can be achieved by the absorption of a photon that consequently excites an electron from the metal into the molecule, leading to charged species on the surface of the (photo)catalyst. More than 20 years ago, Ertl and co-workers already identified this process as an important side pathway to conventional (photo)thermal catalysis. ${ }^{12}$ These reaction mechanisms have been known for many years and can be grouped under the formalism known as desorption induced by electronic transitions (DIET). ${ }^{14,15}$ In these type of processes, the charge carrier transfer 
process to form the transient ion occurs in a non-thermal regime - far from equilibrium within femtosecond timescales after light absorption at the catalyst-molecule interface. ${ }^{16}$ This is shown in Figure 2 with the red arrow that connects the GS and ES. The probability of this electronic transition depends on many factors such as: the energy of the incoming photon, the absorption cross-section of the metal-molecule system, the energy of the generated carrier once at the surface, the energy and density of available electronic states in the system, among others. ${ }^{17}$ In recent years, the possibility of performing this type of reactions by absorbing a plasmon - more than a photon - has impacted our understanding of the photochemical reactivity of molecules adsorbed onto metal (plasmonic) surfaces. ${ }^{3-5}$ Unexplored reaction pathways have been revealed using plasmons excited at continuous visible wavelengths, also revitalizing the idea of sunlight into chemical energy conversion. ${ }^{13,18-}$ ${ }^{21}$ However, there are many different ways in which a plasmonic photocatalyst can help surfing the PES of a chemical reaction.

\section{How can a plasmonic catalyst help?}

When light interacts with metallic NP, part of its energy is transferred to the kinetic motion of free electrons. The quasi-particle associated to the electron oscillation coupled to electromagnetic radiation is called plasmon-polariton (PP) and carries the energy of the incoming photon. A PP can either decay radiatively or be absorbed. Absorption generates a cascade of phenomena that can ultimately be beneficial for chemical transformation of nearby molecules, as schematized in Figure 3. A PP can decay through chemical interface damping directly exciting a carrier from the metal to the molecule. ${ }^{22,23}$ Alternatively, it can decay exciting an electron-hole pair in the NP through different mechanisms, ${ }^{24}$ namely: phonon-assisted absorption, interband absorption, electron-electron scattering-assisted absorption or surface collision-assisted absorption (Landau damping). Each mechanism generates carriers with different energy, spatial and angular distribution and can be assigned with an absorption cross section $\sigma_{p h}, \sigma_{i b}, \sigma_{e e}$ and $\sigma_{s c}$ respectively. The generated energetic carriers scatter with other carriers in the material at a collision rates $\gamma_{e-e}$ and $\gamma_{h-h}$ spreading the energy into a Fermi-Dirac distribution. Carrier-phonon collisions at a rates $\gamma_{e-p h}$ and $\gamma_{h-p h}$ transfer the energy to energetic phonons that will in turn establish a Bose-Einstein distribution through phonon-phonon scattering $\gamma_{p h-p h}$. The phonons interact with the environment releasing heat and increasing the temperature according to different coupling constants such as the thermal conductivity of the metal $\kappa_{N P}$, the medium $\kappa_{m}$ or the Kapitza Resistance $R_{K}$ of the interface. It is important to remark that in the case of pulsed illumination all these processes occur sequentially in the described order. However, for continuous wave (CW) illumination, the processes occur simultaneously and all these physical phenomena coexist. A NP under CW illumination reaches a stationary out-of-equilibrium state holding non-thermal carriers as well as a thermal Fermi-Dirac distribution of carriers and a BoseEinstein distribution of phonons. ${ }^{25}$ As such, the temperatures associated to the electrons, the lattice and the environment (nearby molecules) can be different. ${ }^{26}$ 




Figure 3: The plasmonic equalizer. Manipulating the coupling constants between photons, electrons and phonons allows to tune the relative weight and energy distribution of each plasmon-derived phenomenon. This can alter the reaction pathway by selecting different channels to travel along the A-PES. This is highlighted in the inset as a new coordinate system of the A-PES for a plasmonic photocatalytic reaction.

Interestingly, the stationary state can be engineered to favour the preponderance of one phenomenon over the others, as illustrated in Figure 3. By manipulating the coupling constants between the processes named in the previous paragraph (depicted with knobs in Figure 3), the relative weight of each phenomena can be tuned. For example, Illuminating with wavelengths matching the interband transition of the material will favor carriers generated from interband direct absorption, while illuminating at the plasmon resonance will increase the relative weight of surface damping processes and its corresponding carriers. Materials with reduced electron mean free path will reduce the number of non-thermal carriers and a medium with high thermal conductivity will equalize lattice and medium temperatures.

Let's now reunite the molecule and the photocatalyst worlds. When a molecule is adsorbed to a surface, new hybridized metal-(adsorbed) molecule states are formed, i.e. a new PES for the adsorbed molecule arises (A-PES), different than the free molecule one. It is on this specific A-PES that the PP operates as a versatile supplier of energy quanta ranging from a thermal phonon ( $\cong K_{B} T \cong 0.025 \mathrm{eV}$, with $\mathrm{T}$ the temperature and $K_{B}$ Boltzmann factor) to a hot electron with the energy of the absorbed photon ( $\cong 2-3 \mathrm{eV}$, for the optical regime). Furthermore, the momentum, charge and spin (boson or fermion) of the quanta can be different. This can affect reactivity of molecules in several ways. It can help the molecule to overcome the activation barrier of the A-PES, drive the molecule into a new excited state of the A-PES modifying the reaction pathway, displace the reaction along a given reaction coordinate of the A-PES or do a combination of processes.

It is now clear that the overall reactivity and its spatial localization in plasmon-assisted catalysis - i.e. the chemical hot spot - is a convolution of the light, the reactor, the adsorbed molecule and the reaction pathway. Contrary to optical hot spots, there are several energy channels that can be exploited to boost a chemical reaction ${ }^{19,27,28}$ or to modify reaction 
pathways. ${ }^{20}$ In the following, we review our efforts to understand, map and control chemical reactivity at the single plasmonic particle level. ${ }^{29}$

\section{Discussion}

\section{Spatially-resolved chemistry on hot-electron-driven reactions}

The spatial distribution of reactivity in a catalyst under thermal-driven conditions (i.e. in dark conditions) depends on the magnitude of the RLS activation barrier at each particular point of space. However, in this section, we will focus on reactions driven by non-thermal hotcarriers, as they are appealing for nanoscale localization of reactivity. Given the rapid loss of energy of carriers in systems with high collision rates, their reactivity decreases with increasing distance from where they are generated. Exploiting this idea allows to manipulate the sub-particle spatial distribution of reactivity by determining spots where hot-carriers are preferentially generated.

We began by studying the hot-electron-driven reduction from 4-nitrothiophenol (4-NTP) to 4-aminothiophenol (4-ATP) ${ }^{30}$ on Ag Bow-tie (AgBT) antennas, as schematized in Figure 4a. The surface of AgBT was initially covered with a self-assembled monolayer (SAM) of 4-NTP. After irradiation, $15 \mathrm{~nm}$ Au NPs functionalized with 11-mercaptoundecanoic acid were used as reporters of the reaction. Specific binding between 4-ATP and Au NPs reveals the regions where the plasmon-assisted reaction occurred, as shown in Figure 4b. This constitutes an experimental realization of nano-localized chemistry exploiting plasmonic chemical hot spots.

Another appealing approach to identify chemical hot spots is to image the chemical activity of plasmonic interfaces using super-resolution fluorescence nanoscopy techniques. ${ }^{31,32}$ 2,9,3336 Employing a metallic-DNA PAINT (Point Accumulation for Imaging in Nanoscale Topography) technique (m-PAINT) it has been possible to map the hot-electron induced displacement and re-functionalization of capping molecules from metallic nanostructures. ${ }^{31}$ DNA-PAINT employs transient binding of short fluorescently labeled oligonucleotides ("imager strands") to complementary single stranded DNA oligonucleotides ("docking strands"), which chemically anchored to the NPs through a thiol bond. Figure 4c (i) shows a super-resolution image of the Au nanorods using m-PAINT.

Plasmon-induced selective desorption of the thiolated molecules was achieved by illumination with a horizontally polarized femtosecond laser. Hot-electrons were produced only in the resonant rod as shown in Figure 4c (ii). The uncapped Au surface was then refunctionalized with a different thiolated-DNA SAM, complementary to a second fluorophore, as shown in Figure 4c (iii). Alternatively, the illumination wavelength can also manipulate the spatial distribution of hot electrons. This is exemplified in Figure $4 \mathrm{~d}$ for a Fano resonance system where changing the wavelength $\left(\lambda_{1}, \lambda_{2}\right)$ leads to selective excitation of different regions. ${ }^{32}$ Furthermore, the reactivity of the photocatalyst can be tuned within a single NP, as shown in Figure $4 \mathrm{e} .{ }^{31}$ The hot-electron-induced reaction presents a clear spatial selectivity according to the incident polarization of light, following the distribution of the absorbed power. ${ }^{19,37}$ 


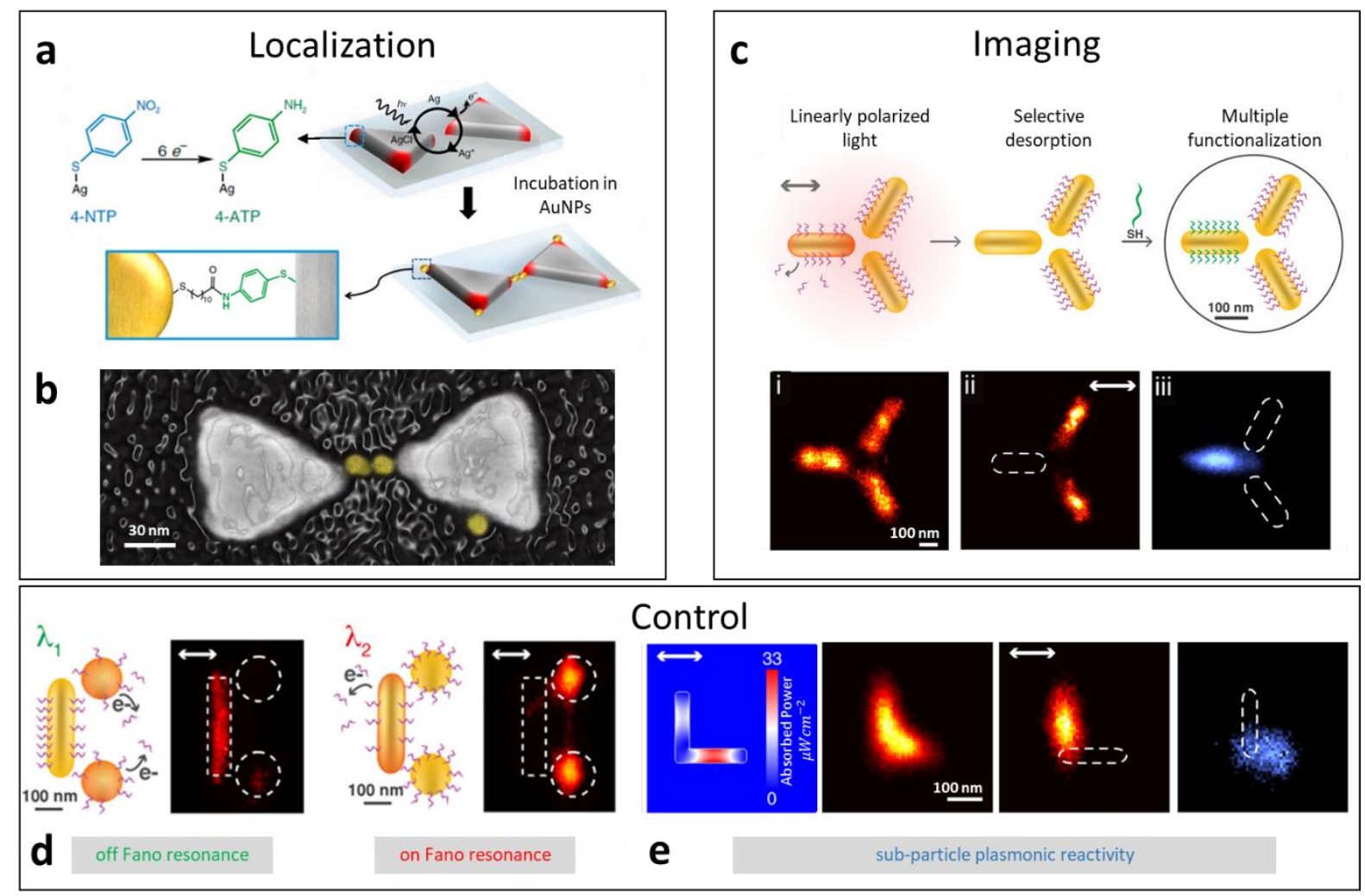

Figure 4: Localizing, imaging and controlling hot-electron-induced processes. Spatial-resolved chemistry can be achieved with hot-electrons. This concept can be further extended to sub-particle local reactivity. Figures adapted with permission from Nature Publishing Group and American Chemical Society: $(a-b)^{8}(c, e)^{31}(d)^{32}$

Further examples of sub-particle control of reactivity are shown in Figure 5, where we follow the hot-electrons reactions for different irradiation powers (a) or times (b). The possibility to tune spatially the chemical composition within plasmonic NPs could open new routes for colloidal synthesis, where the spatial distribution and density of ligands play a critical role. ${ }^{38,39}$ It could also aid in the fabrication of a new generation of plasmonic photocatalysts by selectively positioning molecules/catalyst in highly reactive areas.

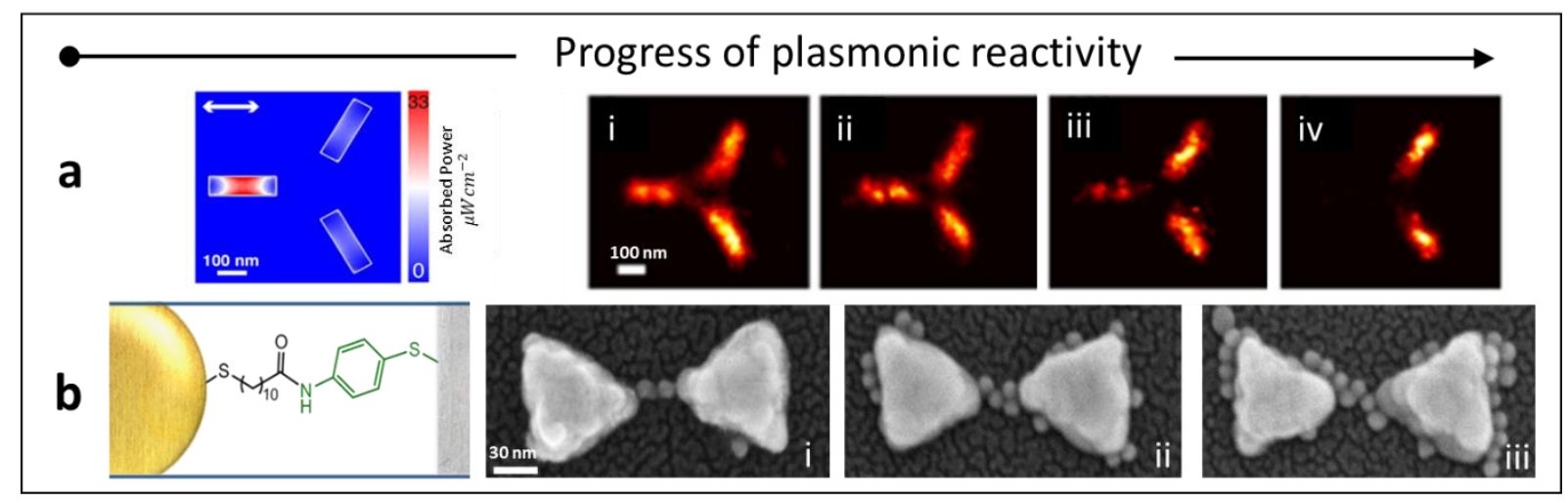

Figure 5: Snapshots of chemical transformations at different illumination powers (a) and times (b) for the hotelectron driven processes described in figure 4. Figures adapted with permission from American Chemical Society and Nature Publishing Group: $(a)^{31}(b)^{8}$ 


\section{Tuning absorption to modify properties of hot carriers}

One of the key questions in plasmon-assisted catalysis is to determine the effective energy contribution from a plasmon-generated carrier to a chemical transformation. Halas et. al ${ }^{19}$ and Jain et al ${ }^{40}$ have studied activation energies in plasmonic catalysis finding that they depend on the incident laser power and the excitation wavelength. These results point towards the idea that reaction pathways can be altered by changing the illumination wavelength. Each wavelength will have a distinctive mechanism of PP decay leading to generated hot carriers with different properties that will in turn produce a different plasmon relaxation cascade. Wavelength is therefore a degree of freedom to manipulate mechanistic details of the reactions. ${ }^{18}$

We have recently presented a method to measure the effective energy contribution from plasmons to a chemical reaction. ${ }^{41}$ We combined dark-field microscopy with an electrochemical set-up to study a plasmonic hot-hole-driven reaction under in operando conditions at the single particle level. The electro-photo-polymerization of aniline on $80 \mathrm{~nm}$ Au nanospheres (AuNS) was followed under CW illumination. To decouple wavelength and intensity effects, the role of the excitation wavelength was studied keeping the absorbed photon flux constant.

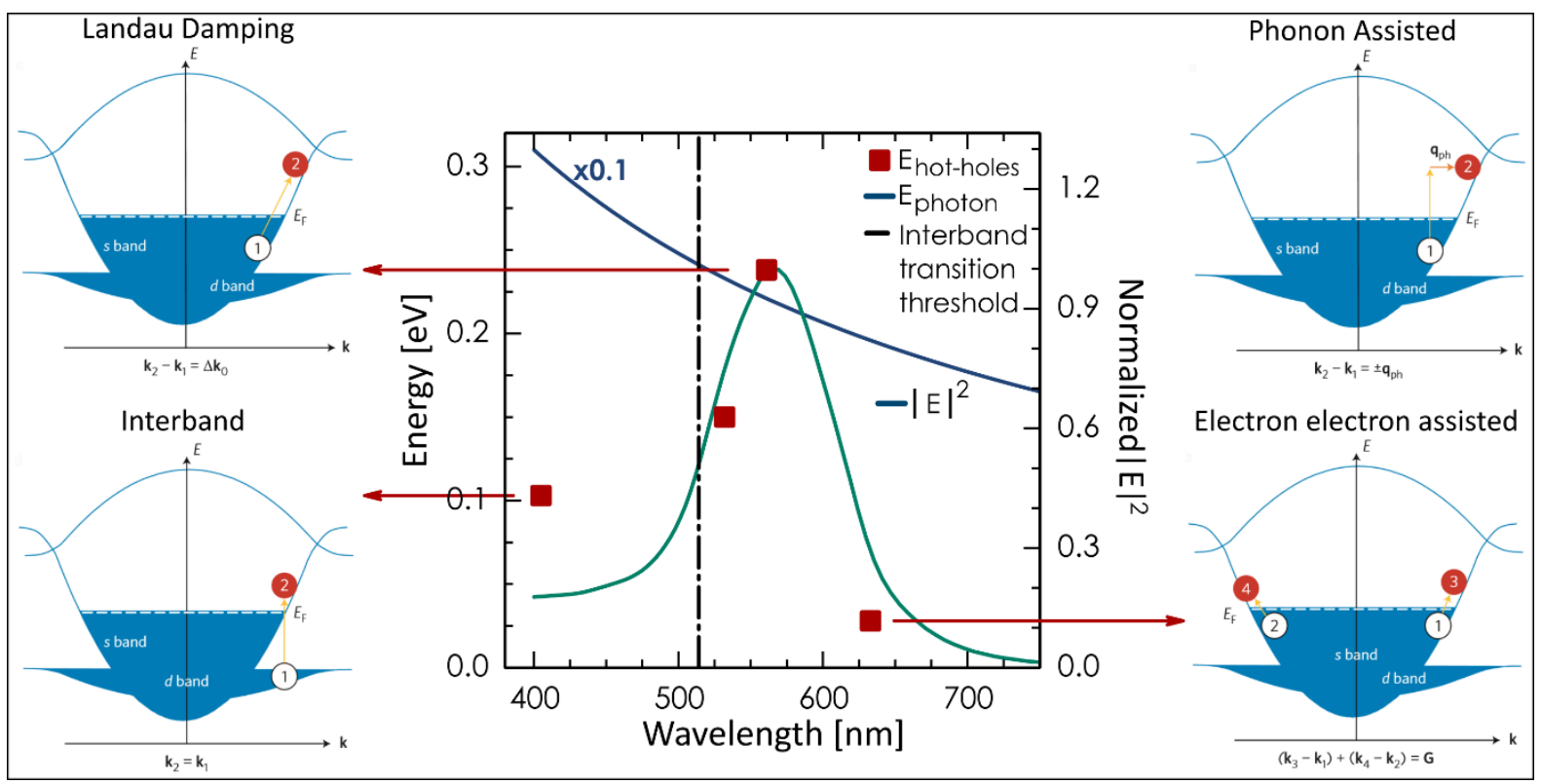

Figure 6: Hot holes energy versus wavelength (red squares, left axis). Energy of the incident photons versus wavelength (blue solid curve, left axis). Note that the data has been divided by a factor of 10 for proper comparison. Normalized integral of the square of the electric field $|E|^{2}$ inside the AuNS (green curve, right axis). Vertical dashed line indicates the threshold wavelength for interband excitations. Red arrows points toward the presumably predominant absorption mechanism at each wavelength. Experimental data adapted with the permission from American Chemical Society. ${ }^{41}$ Absorption mechanisms adapted with permission from Nature Publishing Group. ${ }^{42}$

Figure 6 shows the hot holes energy contribution to the reaction and the energy of the incident photons. Comparison with the normalized integral of the square of the electric field $|E|^{2}$ inside the NP indicates that the maximum effective energy is achieved at the LSPR. These results indicate that the absorption mechanisms determine the cascade of plasmon relaxation 
that ultimately determines the effective energy of the transferred to the molecule. At 405 $\mathrm{nm}$, where interband absorption dominates, the effective energy transferred by d-holes is less than half of the maximum at $561 \mathrm{~nm}$ where Landau damping dominates. ${ }^{41}$ Even if the energy of $d$-holes is high, they have a small mobility, rendering them difficult to be accessed by the molecules. ${ }^{43}$ On the other hand, holes generated by Landau damping are closer to the surface and therefore can lead to more reactive carriers.

A similar approach was implemented by Willets et al, who quantified the thermalized hot carrier energy distribution generated at $\mathrm{Au} / \mathrm{TiO}_{2}$ nanostructures using wavelength-dependent scanning electrochemical microscopy and a series of molecular probes with different redox potentials. ${ }^{44}$ These experiments provide examples of how the properties of the generated carriers can be tuned by favoring different plasmon decay mechanisms, influencing the ultimate reactivity of plasmonic reactors.

\section{Manipulating hot-carrier relaxation}

The typical timescale for electron-electron (e-e) interaction and electron-phonon (e-ph) interaction are in the tens of femtoseconds and a few picoseconds range, respectively, leading to a fast energy redistribution from a single hot electron-hole pair to other carriers in the material. This can be detrimental for the overall reactivity of the system, as carriers with higher energy can have access to more energetic molecular states. It is therefore appealing to study, control and manipulate the coupling between carriers and phonons. An interesting example was demonstrated by Ortolani et $a l^{45}$ in nanoporous gold films. They showed that the relaxation times between electrons and the lattice scales with the metal filling factor, indicating that the energy of hot electrons in porous gold films takes longer times to dissipate, increasing their probability to be transferred to molecular states.

Interfacing the metal with a different material can also dramatically alter hot-carrier relaxation. ${ }^{46,47} \mathrm{New}$ electronic states are created that can capture the carriers before their decay, keeping carriers 'warm' for longer times. In particular, metal-semiconductor interfaces produce Schottky barriers with an energy determined by the difference between the Fermi level in the metal and the conduction band of the semiconductor. ${ }^{35-48-50}$ This barrier sets an energy threshold for the transfer of carriers. However, different from metals, the conduction band of semiconductors is empty, thus highly suppressing e-e scattering once the hot-carrier has been transferred. For example, using X-ray absorption spectroscopy (XAS), Cushing et al investigated $\mathrm{Au} @ \mathrm{TiO}_{2}$ particles, finding that the electrons transferred from the metal remain non-thermal in the $\sim 10 \mathrm{~nm} \mathrm{TiO} 2$ shell. ${ }^{51}$ Moreover, altering the semiconductor composition can influence even further the trapping and recombination capabilities of the generated carriers. Variation of oxygen content in $\mathrm{TiO}_{2-x}$ films allows tunability of free carrier densities at the surface of the material by an order of magnitude. This leads to the presence of out-ofequilibrium hot-carriers at the external surface of the material, readily available to participate in surface chemical reactions. ${ }^{52}$ We have indeed recently sensed an enhanced charge transfer pathway in $\mathrm{Au} / \mathrm{TiO}_{2}$ composites due to the presence of oxygen vacancies in the semiconductor (Figure 7a). This produces an extra enhancement in the Raman scattering signal of a wide range of molecules, as exemplified for Rhodamine $6 \mathrm{G}$ in Figure $7 \mathrm{~b}$. 
An interesting method to study the dynamics of hot carriers are ultrafast pump-probe reflectivity measurements. ${ }^{53}$ Photo-generated carriers introduce refractive index changes that result in a reflectance variation. Figure 7c shows the estimated electron occupancy generated on an illuminated $30 \mathrm{~nm}$ gold film. Changing the angle of incidence of the light on the film modifies the properties of the excited plasmon, influencing the energy distribution of the generated carriers. This could provide an extra degree of freedom for controlling the properties of the generated carriers in plasmonic systems. Figure $7 \mathrm{~d}$ illustrates our pumpprobe study of the Schottky junction on single Au-Clad Si nanodisk, where we observed extended lifetimes of tens of picoseconds attributed to the band filling by free carriers. ${ }^{54}$ Further ultrafast studies including molecules as receptors of the hot-carriers could open new doors for the timescale management of these processes.



Figure 7. (a) Photo-induced enhanced Raman spectroscopy (PIERS), where occupied vacancy states in $\mathrm{TiO}_{2}$ favor charge transfer and Raman vibrational modes. (b) Experimental spectra of Rhodamine 6G molecules with trapassistance. (c) Differential reflectivity and derived electron occupancy changes for the $30 \mathrm{~nm}$ gold film at normal incidence. (d) Experimental differential reflectance of a gold-clad silicon disk. Figures adapted with permission from American Chemical Society and Nature Publishing Group: $(a-b)^{55}(c)^{53}(d)^{54}$

\section{Phonons and nanoscale heating}

Highly non-thermal and thermalized carriers efficiently excite phonons in the host lattice. This dynamics is particularly rich in small structures when illuminated by ultrashort laser pulses, whose physical boundaries define additional confining conditions for the launched mechanical modes, affecting mainly those of long wavelength compared to the dimensions of the new imposed boundaries. ${ }^{56}$ From a highly non-equilibrium condition of carriers, this quantization of strongly-dispersive acoustic phonons close to the center of the Brillouin zone leads to another highly non-equilibrium situation per se, where the phonon energy distribution now deviates significantly from the characteristic Bose-Einstein distribution at a 
given temperature, leading to further relaxation via phonon-phonon scattering processes. ${ }^{57}$ The final thermal distribution results in a heated particle, which has been demonstrated useful for thermal catalysis ${ }^{58}$ and whose equilibrium temperature is dependent on the system's parameters such as the substrate material. ${ }^{59}$

Control of the generation of coherent acoustic phonons following the cascade of events of plasmon relaxation was achieved by changing the boundary conditions of the resonators, such as, for instance, adding mechanical constraints to metallic nanostructures. ${ }^{60} \mathrm{~A}$ high degree of control and tunability of mechanical modes was obtained, as shown in Figure 8a, where the extensional mode of the Au rod could be tuned by more than $100 \%$ by changing the height of dielectric constraints attached to its ends. These coherent vibrations have welldefined displacement profiles depending on the NP geometry, and such localized displacements, when properly engineered, could play a role in catalysis by enhancing the desorption of reaction products, releasing active sites for reactants.

Also interesting is the investigation of the decay of coherent vibrations from single metallic nanostructures to the underlying substrate through the emission of acoustic waves. ${ }^{61} \mathrm{~A}$ significant emission of SAWs has been obtained by observing how they modulate the LSPR of a second NP positioned at large distances when compared to the average sub-nanometric displacements of source modes (Figure 8b). Besides, this modulation has been shown to occur at the frequency of the main extensional mode of the Au rod source. Apart from the exquisite sensitivity of plasmonic structures to mechanical vibrations, these sub-diffraction sources of SAWs of well-defined frequency could prove to be an invaluable tool in the study of nonequilibrium phonon relaxation and nonlinear phononics. ${ }^{62,63}$

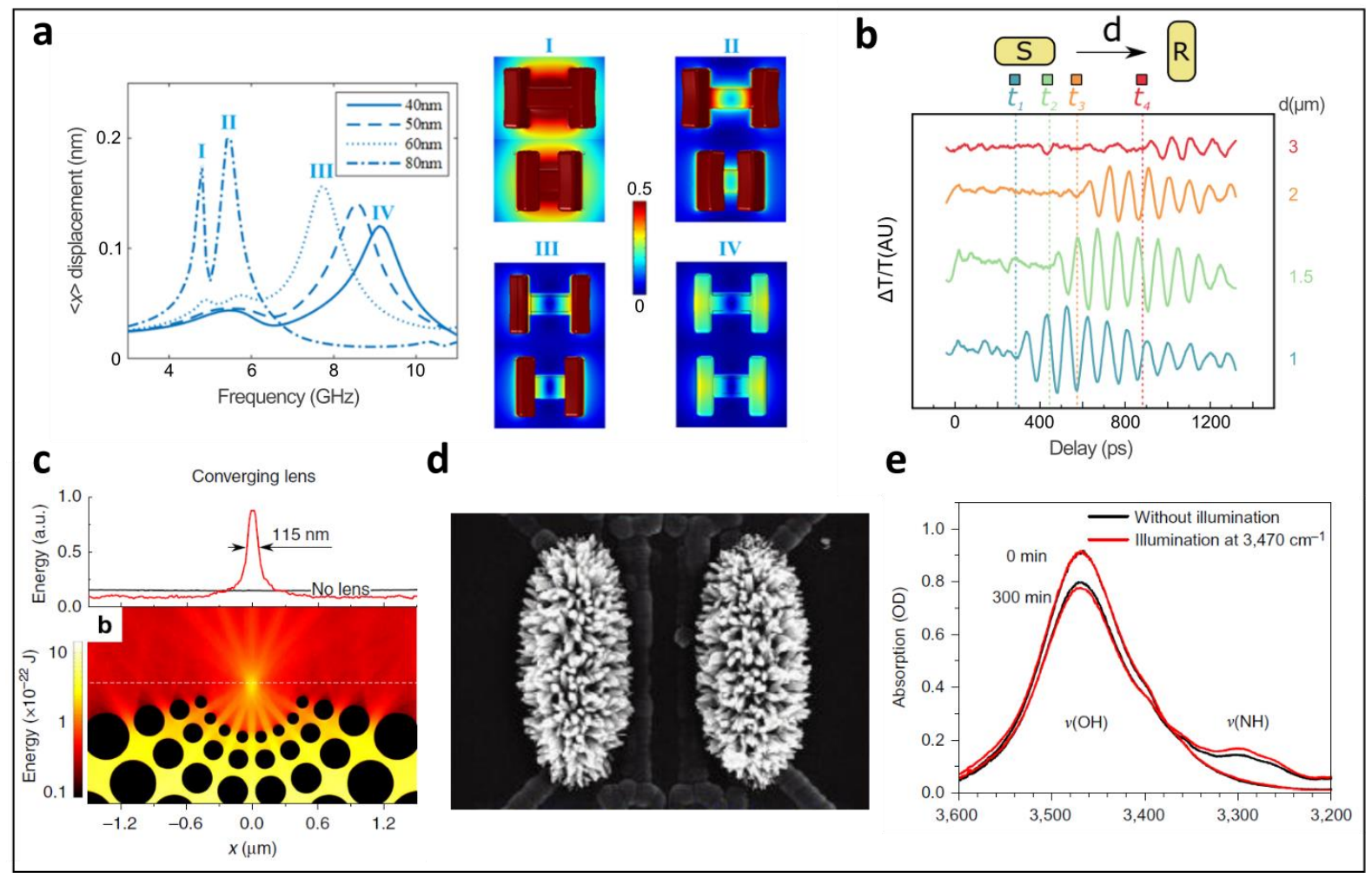


Figure 8: (a) Tuning of plasmonic rod coherent phonon mode by dielectric constraints of different height. ${ }^{60}(\mathrm{~b})$ : Acoustic far-field detection of SAWs generated from coherent phonon mode decay. ${ }^{61}$ (c): Ballistic phonons focusing using thin-membrane phononic crystals. ${ }^{64}(\mathrm{~d})$ : Chemical reaction control at the nanoscale from selective joule heating of nanowires. ${ }^{65}$ (e) Increase in chemical reaction rate by ultrafast IR pumping of vibrations participating in the reaction coordinate. ${ }^{66}$ Figures adapted with permission from Nature Publishing Group, American Physical Society and American Chemical Society: (a) ${ }^{60}$ (b) ${ }^{61}$ (c) ${ }^{64}$ (d) ${ }^{65}$ (e) ${ }^{66}$

Another degree of control of the relaxation of optically pumped metallic structures regards the far-field directionality in the propagation of mechanical vibrations, as shown for ballistic phonons in silicon membranes with arrays of holes, ${ }^{64}$ enabling heat guiding and focusing at the nanoscale (Figure 8c). These could allow temporal and spatial control of chemical reactions, as demonstrated for the localized hydrothermal synthesis of $\mathrm{ZnO}$ over electricallybiased $\mathrm{Cr}$ nanowires subject to Joule heating (Figure $8 \mathrm{~d}$ ). ${ }^{65}$ By positioning a nanostructure at the focus of the incoming vibrations, the coupling to its mechanical modes and subsequent energy dissipation could provide selective and controlled heating of the nanostructure. This may enables, for example, the study of photothermal effects in nanocatalysts, excluding the contributions from optically pumped non-thermal carriers.

An exciting prospect in controlling chemical reactions involves the direct coupling between the generated phonons and vibrational modes of molecules that participate in the reaction coordinate, analogous as proposed by using hot-carriers and mid-infrared surface plasmons. ${ }^{5,19,26}$ As recently demonstrated, ${ }^{66}$ IR pumping at the vibrational frequency of a given chemical bond - involved in the reaction pathway - has shown an increased rate of product formation (Figure 8e). An enhanced excitation of desired molecular vibrations could be also provided by the coupling of optic phonons with light in hybrid light-matter excitations termed surface phonon polaritons (SPhP), ${ }^{67}$ where now the coupling and pumping of specific molecular vibrational modes can be mediated by the polariton moiety of the SPhP. High-Q factor modes of polar dielectric resonators in the mid-IR have shown great potential for chemical sensing. ${ }^{68,69}$ In this scenario, chemical reaction rates can be further enhanced, similarly as obtained by Stensitzki et al using far-field illumination for a homogeneous photocatalytic process. ${ }^{66}$ This could open new pathways for controlling the selectivity of chemical reactions with nano-optics, analogously to what has been recently shown for strongly coupled systems of molecules into optical cavities. ${ }^{70-72}$

\section{Outlook and Perspectives}

Plasmon excitation and decay in metal NPs possess many useful energy channels to explore the ground and excited PESs of a chemical reaction. ${ }^{13}$ The dominant plasmon-derived mechanism contributing most to the reaction might strongly depend on the RLS of the molecule-catalyst system under study. Our single particle experiments ${ }^{29}$ have shown that in some cases the hot-carriers effect ${ }^{31,41}$ or the near-field contribution ${ }^{73}$ can dominate over the thermal pathway. However, one of our current bottlenecks to make wiser use of these phenomena is the lack of computational methods able to predict the energy landscape of adsorbed molecules, especially in the excited state. ${ }^{74}$ For this reason, the real opportunities for non-thermal mediated reactions - where the excited state of the system needs to be 
accessed - are still mostly unknown..$^{23,74,75}$ As such, it might be possible that new materials not considered catalytic up to now for the ground PES of a chemical reaction - may present an interesting excited state energy landscape for the adsorbed molecules The combination of these excited-state photocatalytic materials with plasmonic NPs may open new routes for the field. Indeed, plasmonic hybrid nanostructures are an appealing route for combining material properties to enhance chemical reactivity. The combination of traditional (ground PES) catalytic and plasmonic materials has already shown promising ways of merging heterogeneous catalysis and plasmonics. ${ }^{76,77}$ In the same line, hybrid plasmonicsemiconductor photocatalysts could help extending the lifetime of hot-carriers, ${ }^{47,48}$ diminishing electron and phonon scattering effects. ${ }^{78,79}$

The possibility to navigate a reaction pathway different than the thermal one may also be achieved in the ground PES by using plasmons derived phenomena. Phonons or acoustic waves could be employed for vibrational excitation of a given chemical bond leading to selective formation of products. However, independently of the PES that we are navigating, we still need temperature to overcome the activation barriers of the reaction. For this reason, there is a necessity to develop new thermometry and heat managing techniques that allow the control and characterization of temperature fields at the single catalyst level. Advances in this area could help to better rationalize and enhance the desired effect of temperature.

Photons, electrons and phonons can be channeled and manipulated to create plasmonic chemical hotspots. Independent on the exact mechanism operating at plasmonic interfaces, great contributions to the field photocatalysis have been made in recent years. Plasmons have opened access to enhance and control chemical reactivity with $\mathrm{CW}$ illumination in the visible range, a fundamental requisite for sunlight photocatalysis. Further understanding and control of these processes could hopefully impact in potential industrial applications of photocatalysis, which so far have remain elusive.

\section{Author Information}

\section{Corresponding Author}

*E-mail: Emiliano.Cortes@Imu.de

\section{Funding}

The authors acknowledge the Deutsche Forschungsgemeinschaft (Clusters of Excellence: Nanolnitiative Munich and e-Conversion), the Bavarian Solar Energies Go Hybrid (SolTech) programme and the Center for NanoScience (CeNS). J. Gargiulo acknowledges the European Commission for a Marie-Sklodowska-Curie action, Y. Li the European Union's Framework Programme for Research and Innovation Horizon 2020 (2014-2020) under the Marie Skłodowska-Curie Grant Agreement No. 754388 and the framework of the German Excellence Initiative (No. ZUK22), and E. Cortés the ERC starting grant 802989 CATALIGHT.

\section{Notes}


The authors declare no competing financial interest. The authors acknowledge Ms. Lin Nan for assisting in the figures design.

\section{Biographical Information}

Julian Gargiulo received his Ph.D. degree in Physics at the Universidad de Buenos Aires (UBA). He was a research associate at Imperial College London (ICL) and is currently a Marie Skłodowska-Curie (MSC) research fellow at Ludwig-Maximilians-Universität München (LMU).

Rodrigo Berté received his Ph.D. degree in Physics at the ICL supported by a Science Without Borders fellowship from the Capes Foundation/Brazil. He is currently a research associate at LMU.

Yi Li received his Ph.D. degree in Electrical Engineering at the KU Leuven and IMEC, Belgium. He has been a research associate at ICL and he is currently an LMU research fellow.

Stefan A. Maier received his Ph.D. degree at the California Institute of Technology and has worked at the University of Bath, ICL, and holds now the Chair in Hybrid Nanosystems at the Faculty of Physics, LMU.

Emiliano Cortés received his Ph.D. degree at Universidad de La Plata, Argentina. He was one of the founders of Nanodetection, a start-up company based on plasmonic sensing. He was a postdoc at UBA and MSC research fellow at ICL, UK. He has been recently awarded with the ERC Starting Grant from the European Commission for his project CATALIGHT and he is now Associate Professor at the Faculty of Physics, LMU Germany.

\section{References}

(1) Le Ru, E.; Etchegoin, P. Principles of Surface-Enhanced Raman Spectroscopy: And Related Plasmonic Effects; Elsevier, 2008.

(2) Mack, D. L.; Cortés, E.; Giannini, V.; Török, P.; Roschuk, T.; Maier, S. A. Decoupling Absorption and Emission Processes in Super-Resolution Localization of Emitters in a Plasmonic Hotspot. Nat. Commun. 2017, 8, 14513.

(3) Aslam, U.; Rao, V. G.; Chavez, S.; Linic, S. Catalytic Conversion of Solar to Chemical Energy on Plasmonic Metal Nanostructures. Nat. Catal. 2018, 1, 656-665.

(4) Zhang, Y.; He, S.; Guo, W.; Hu, Y.; Huang, J.; Mulcahy, J. R.; Wei, W. D. Surface-PlasmonDriven Hot Electron Photochemistry. Chem. Rev. 2018, 118, 2927-2954.

(5) Zhan, C.; Chen, X.-J.; Yi, J.; Li, J.-F.; Wu, D.-Y.; Tian, Z.-Q. From Plasmon-Enhanced Molecular Spectroscopy to Plasmon-Mediated Chemical Reactions. Nat. Rev. Chem. 2018, 2, 216-230.

(6) Brongersma, M. L.; Halas, N. J.; Nordlander, P. Plasmon-Induced Hot Carrier Science and Technology. Nat. Nanotechnol. 2015, 10, 25-34.

(7) Christopher, P.; Xin, H.; Linic, S. Visible-Light-Enhanced Catalytic Oxidation Reactions on Plasmonic Silver Nanostructures. Nat. Chem. 2011, 3, 467-472.

(8) Cortés, E.; Xie, W.; Cambiasso, J.; Jermyn, A. S.; Sundararaman, R.; Narang, P.; Schlücker, S.; 
Maier, S. A. Plasmonic Hot Electron Transport Drives Nano-Localized Chemistry. Nat. Commun. 2017, 8, 14880.

(9) Chen, T.; Dong, B.; Chen, K.; Zhao, F.; Cheng, X.; Ma, C.; Lee, S.; Zhang, P.; Kang, S. H.; Ha, J. W.; Xu, W.; Fang, N. Optical Super-Resolution Imaging of Surface Reactions. Chem. Rev. 2017, $117,7510-7537$.

(10) Andoy, N. M.; Zhou, X.; Choudhary, E.; Shen, H.; Liu, G.; Chen, P. Single-Molecule Catalysis Mapping Quantifies Site-Specific Activity and Uncovers Radial Activity Gradient on Single 2D Nanocrystals. J. Am. Chem. Soc. 2013, 135, 1845-1852.

(11) Ertl, G. Reactions at Surfaces: From Atoms to Complexity (Nobel Lecture). Angew. Chemie Int. Ed. 2008, 47, 3524-3535.

(12) Bonn, M.; Funk, S.; Hess, C.; Denzler, D. N.; Stampfl, C.; Scheffler, M.; Wolf, M.; Ertl, G. Phonon- versus Electron-Mediated Desorption and Oxidation of $\mathrm{CO}$ on $\mathrm{Ru}(0001)$. Science 1999, 285, 1042-1045.

(13) Cortés, E. Activating Plasmonic Chemistry. Science 2018, 362, 28-29.

(14) Menzel, D.; Gomer, R. Desorption from Surfaces by Slow-Electron Impact. J. Chem. Phys. 1964, 40, 1164-1165.

(15) Redhead, P. A. Interaction of Slow Electrons with Chemisorbed Oxygen. Can. J. Phys. 1964, 42, 886-905.

(16) Ho, W. Reactions at Metal Surfaces Induced by Femtosecond Lasers, Tunneling Electrons, and Heating. J. Phys. Chem. 1996, 100, 13050-13060.

(17) Cortés, E. Efficiency and Bond Selectivity in Plasmon-Induced Photochemistry. Adv. Opt. Mater. 2017, 5, 1700191.

(18) Boerigter, C.; Campana, R.; Morabito, M.; Linic, S. Evidence and Implications of Direct Charge Excitation as the Dominant Mechanism in Plasmon-Mediated Photocatalysis. Nat. Commun. 2016, 7, 10545.

(19) Zhou, L.; Swearer, D. F.; Zhang, C.; Robatjazi, H.; Zhao, H.; Henderson, L.; Dong, L.; Christopher, P.; Carter, E. A.; Nordlander, P.; Halas, N. J. Quantifying Hot Carrier and Thermal Contributions in Plasmonic Photocatalysis. Science 2018, 362, 69-72.

(20) Zhang, X.; Li, X.; Zhang, D.; Su, N. Q.; Yang, W.; Everitt, H. O.; Liu, J. Product Selectivity in Plasmonic Photocatalysis for Carbon Dioxide Hydrogenation. Nat. Commun. 2017, 8, 14542.

(21) Linic, S.; Christopher, P.; Ingram, D. B. Plasmonic-Metal Nanostructures for Efficient Conversion of Solar to Chemical Energy. Nat. Mater. 2011, 10, 911-921.

(22) Wu, K.; Chen, J.; McBride, J. R.; Lian, T. Efficient Hot-Electron Transfer by a Plasmon-Induced Interfacial Charge-Transfer Transition. Science 2015, 349, 632-635.

(23) Kumar, P. V.; Rossi, T. P.; Kuisma, M.; Erhart, P.; Norris, D. J. Direct Hot-Carrier Transfer in Plasmonic Catalysis. Faraday Discuss. 2019, 214, 189-197.

(24) Khurgin, J. B. Hot Carriers Generated by Plasmons: Where Are They Are Generated and Where Do They Go from There? Faraday Discuss. 2019, 214, 35-58.

(25) Sivan, Y.; Un, I. W.; Dubi, Y. Assistance of Metal Nanoparticles in Photocatalysis - Nothing More than a Classical Heat Source. Faraday Discuss. 2019, 214, 215-233.

(26) Boerigter, C.; Aslam, U.; Linic, S. Mechanism of Charge Transfer from Plasmonic 
Nanostructures to Chemically Attached Materials. ACS Nano 2016, 10, 6108-6115.

(27) Li, X.; Zhang, X.; Everitt, H. O.; Liu, J. Light-Induced Thermal Gradients in Ruthenium Catalysts Significantly Enhance Ammonia Production. Nano Lett. 2019, 19, 1706-1711.

(28) Seemala, B.; Therrien, A. J.; Lou, M.; Li, K.; Finzel, J. P.; Qi, J.; Nordlander, P.; Christopher, P. Plasmon-Mediated Catalytic O 2 Dissociation on Ag Nanostructures: Hot Electrons or Near Fields? ACS Energy Lett. 2019, 4, 1803-1809.

(29) Simoncelli, S.; Pensa, E. L.; Brick, T.; Gargiulo, J.; Lauri, A.; Cambiasso, J.; Li, Y.; Maier, S. A.; Cortés, E. Monitoring Plasmonic Hot-Carrier Chemical Reactions at the Single Particle Level. Faraday Discuss. 2019, 73-87.

(30) Xie, W.; Schlücker, S. Hot Electron-Induced Reduction of Small Molecules on Photorecycling Metal Surfaces. Nat. Commun. 2015, 6, 7570.

(31) Simoncelli, S.; Li, Y.; Cortés, E.; Maier, S. A. Nanoscale Control of Molecular Self-Assembly Induced by Plasmonic Hot-Electron Dynamics. ACS Nano 2018, 12, 2184-2192.

(32) Simoncelli, S.; Li, Y.; Cortés, E.; Maier, S. A. Imaging Plasmon Hybridization of Fano Resonances via Hot-Electron-Mediated Absorption Mapping. Nano Lett. 2018, 18, 34003406.

(33) Jang, Y. J.; Chung, K.; Lee, J. S.; Choi, C. H.; Lim, J. W.; Kim, D. H. Plasmonic Hot Carriers Imaging: Promise and Outlook. ACS Photonics 2018, 5, 4711-4723.

(34) Fang, Y.; Wang, H.; Yu, H.; Liu, X.; Wang, W.; Chen, H. Y.; Tao, N. J. Plasmonic Imaging of Electrochemical Reactions of Single Nanoparticles. Acc. Chem. Res. 2016, 49, 2614-2624.

(35) Willets, K. A.; Wilson, A. J.; Sundaresan, V.; Joshi, P. B. Super-Resolution Imaging and Plasmonics. Chem. Rev. 2017, 117, 7538-7582.

(36) Cortés, E.; Huidobro, P. A.; Sinclair, H. G.; Guldbrand, S.; Peveler, W. J.; Davies, T.; Parrinello, S.; Görlitz, F.; Dunsby, C.; Neil, M. A. A.; Sivan, Y.; Parkin, I. P.; French, P. M. W.; Maier, S. A. Plasmonic Nanoprobes for Stimulated Emission Depletion Nanoscopy. ACS Nano 2016, 10, 10454-10461.

(37) Zhang, J.; Guan, M.; Lischner, J.; Meng, S.; Prezhdo, O. V. Coexistence of Different ChargeTransfer Mechanisms in the Hot-Carrier Dynamics of Hybrid Plasmonic Nanomaterials. Nano Lett. 2019, 19, 3187-3193.

(38) González-Rubio, G.; Díaz-Núñez, P.; Rivera, A.; Prada, A.; Tardajos, G.; González-Izquierdo, J.; Bañares, L.; Llombart, P.; Macdowell, L. G.; Palafox, M. A.; Liz-Marzán, L. M.; Peña-Rodríguez, O.; Guerrero-Martínez, A. Femtosecond Laser Reshaping Yields Gold Nanorods with Ultranarrow Surface Plasmon Resonances. Science 2017, 358, 640-644.

(39) Zhai, Y.; DuChene, J. S.; Wang, Y.-C. C.; Qiu, J.; Johnston-Peck, A. C.; You, B.; Guo, W.; Diciaccio, B.; Qian, K.; Zhao, E. W.; Ooi, F.; Hu, D.; Su, D.; Stach, E. A.; Zhu, Z.; Wei, W. D. Polyvinylpyrrolidone-Induced Anisotropic Growth of Gold Nanoprisms in Plasmon-Driven Synthesis. Nat. Mater. 2016, 15, 889-895.

(40) Kim, Y.; Dumett Torres, D.; Jain, P. K. Activation Energies of Plasmonic Catalysts. Nano Lett. 2016, 16, 3399-3407.

(41) Pensa, E. L.; Gargiulo, J.; Lauri, A.; Schlücker, S.; Cortes, E.; Maier, S. A. Spectral Screening of the Energy of Hot Holes over a Particle Plasmon Resonance. Nano Lett. 2019, 19, 1867-1874.

(42) Khurgin, J. B. How to Deal with the Loss in Plasmonics and Metamaterials. Nat. Nanotechnol. 
$2015,10,2-6$.

(43) Brown, A. M.; Sundararaman, R.; Narang, P.; Goddard, W. A.; Atwater, H. A. Nonradiative Plasmon Decay and Hot Carrier Dynamics: Effects of Phonons, Surfaces, and Geometry. ACS Nano 2016, 10, 957-966.

(44) Yu, Y.; Wijesekara, K. D.; Xi, X.; Willets, K. A. Quantifying Wavelength-Dependent Plasmonic Hot Carrier Energy Distributions at Metal/Semiconductor Interfaces. ACS Nano 2019, 13, 3629-3637.

(45) Ortolani, M.; Brida, D.; de Angelis, F.; Mancini, A.; Budweg, A.; Garoli, D. Pump-Probe Spectroscopy Study of Ultrafast Temperature Dynamics in Nanoporous Gold. Phys. Rev. B 2019, 99, 035435.

(46) Harutyunyan, H.; Martinson, A. B. F.; Rosenmann, D.; Khorashad, L. K.; Besteiro, L. V.; Govorov, A. O.; Wiederrecht, G. P. Anomalous Ultrafast Dynamics of Hot Plasmonic Electrons in Nanostructures with Hot Spots. Nat. Nanotechnol. 2015, 10, 770-774.

(47) Zhan, C.; Wang, Z. Y.; Zhang, X. G.; Chen, X. J.; Huang, Y. F.; Hu, S.; Li, J. F.; Wu, D. Y.; Moskovits, M.; Tian, Z. Q. Interfacial Construction of Plasmonic Nanostructures for the Utilization of the Plasmon-Excited Electrons and Holes. J. Am. Chem. Soc. 2019, 141, 80538057.

(48) Christopher, P.; Moskovits, M. Hot Charge Carrier Transmission from Plasmonic Nanostructures. Annu. Rev. Phys. Chem. 2017, 68, 379-398.

(49) Park, J. Y.; Baker, L. R.; Somorjai, G. a. Role of Hot Electrons and Metal-Oxide Interfaces in Surface Chemistry and Catalytic Reactions. Chem. Rev. 2015, 115, 2781-2817.

(50) Clavero, C. Plasmon-Induced Hot-Electron Generation at Nanoparticle/Metal-Oxide Interfaces for Photovoltaic and Photocatalytic Devices. Nat. Photon. 2014, 8, 95-103.

(51) Cushing, S. K.; Chen, C. J.; Dong, C. L.; Kong, X. T.; Govorov, A. O.; Liu, R. S.; Wu, N. Tunable Nonthermal Distribution of Hot Electrons in a Semiconductor Injected from a Plasmonic Gold Nanostructure. ACS Nano 2018, 12, 7117-7126.

(52) Habib, A.; Florio, F.; Sundararaman, R. Hot Carrier Dynamics in Plasmonic Transition Metal Nitrides. J. Opt. 2018, 20, 064001.

(53) Heilpern, T.; Manjare, M.; Govorov, A. O.; Wiederrecht, G. P.; Gray, S. K.; Harutyunyan, H. Determination of Hot Carrier Energy Distributions from Inversion of Ultrafast Pump-Probe Reflectivity Measurements. Nat. Commun. 2018, 9, 1853.

(54) Grinblat, G.; Berte, R.; Nielsen, M. P. P.; Li, Y.; Oulton, R. F.; Maier, S. A. Sub-20 Fs All-Optical Switching in a Single Au-Clad Si Nanodisk. Nano Lett. 2018, 18, 7896-7900.

(55) Ben-Jaber, S.; Peveler, W. J.; Quesada-Cabrera, R.; Cortés, E.; Sotelo-Vazquez, C.; AbdulKarim, N.; Maier, S. A.; Parkin, I. P. Photo-Induced Enhanced Raman Spectroscopy for Universal Ultra-Trace Detection of Explosives, Pollutants and Biomolecules. Nat. Commun. 2016, 7, 12189.

(56) Crut, A.; Maioli, P.; Del Fatti, N.; Vallée, F. Time-Domain Investigation of the Acoustic Vibrations of Metal Nanoparticles: Size and Encapsulation Effects. Ultrasonics 2015, 56, 98 108.

(57) Ashcroft, N, W.; Mermin, N. D. Solid State Physics; 2014.

(58) Baffou, G.; Quidant, R. Nanoplasmonics for Chemistry. Chem. Soc. Rev. 2014, 43, 3898-3907. 
(59) Gargiulo, J.; Brick, T.; Violi, I. L.; Herrera, F. C.; Shibanuma, T.; Albella, P.; Requejo, F. G.; Cortés, E.; Maier, S. A.; Stefani, F. D. Understanding and Reducing Photothermal Forces for the Fabrication of Au Nanoparticle Dimers by Optical Printing. Nano Lett. 2017, 17, 57475755.

(60) Della Picca, F.; Berte, R.; Rahmani, M.; Albella, P.; Bujjamer, J. M.; Poblet, M.; Cortés, E.; Maier, S. A.; Bragas, A. Tailored Hypersound Generation in Single Plasmonic Nanoantennas. Nano Lett. 2016, 16, 1428-1434.

(61) Berte, R.; Picca, F. Della; Poblet, M.; Li, Y.; Cort, E.; Craster, R. V; Maier, S. A.; Bragas, A. V. Acoustic Far-Field Hypersonic Surface Wave Detection with Single Plasmonic Nanoantennas. Phys. Rev. Lett. 2018, 121, 253902.

(62) von Hoegen, A.; Mankowsky, R.; Fechner, M.; Först, M.; Cavalleri, A. Probing the Interatomic Potential of Solids with Strong-Field Nonlinear Phononics. Nature 2018, 555, 79-82.

(63) Bojahr, A.; Gohlke, M.; Leitenberger, W.; Pudell, J.; Reinhardt, M.; Von Reppert, A.; Roessle, M.; Sander, M.; Gaal, P.; Bargheer, M. Second Harmonic Generation of Nanoscale Phonon Wave Packets. Phys. Rev. Lett. 2015, 115, 195502.

(64) Anufriev, R.; Ramiere, A.; Maire, J.; Nomura, M. Heat Guiding and Focusing Using Ballistic Phonon Transport in Phononic Nanostructures. Nat. Commun. 2017, 8, 155505.

(65) Jin, C. Y.; Li, Z.; Williams, R. S.; Lee, K. C.; Park, I. Localized Temperature and Chemical Reaction Control in Nanoscale Space by Nanowire Array. Nano Lett. 2011, 11, 4818-4825.

(66) Stensitzki, T.; Yang, Y.; Kozich, V.; Ahmed, A. A.; Kössl, F.; Kühn, O.; Heyne, K. Acceleration of a Ground-State Reaction by Selective Femtosecond-Infrared-Laser-Pulse Excitation. Nat. Chem. 2018, 10, 126-131.

(67) Caldwell, J. D.; Lindsay, L.; Giannini, V.; Vurgaftman, I.; Reinecke, T. L.; Maier, S. A.; Glembocki, O. J. Low-Loss, Infrared and Terahertz Nanophotonics Using Surface Phonon Polaritons. Nanophotonics 2015, 4, 44-68.

(68) Berte, R.; Gubbin, C. R.; Wheeler, V. D.; Giles, A. J.; Giannini, V.; Maier, S. A.; De Liberato, S.; Caldwell, J. D. Sub-Nanometer Thin Oxide Film Sensing with Localized Surface Phonon Polaritons. ACS Photonics 2018, 5, 2807-2815.

(69) Autore, M.; Li, P.; Dolado, I.; Alfaro-Mozaz, F. J.; Esteban, R.; Atxabal, A.; Casanova, F.; Hueso, L. E.; Alonso-González, P.; Aizpurua, J.; Nikitin, A. Y.; Vélez, S.; Hillenbrand, R. Boron Nitride Nanoresonators for Phonon-Enhanced Molecular Vibrational Spectroscopy at the Strong Coupling Limit. Light Sci. Appl. 2018, 7, 17172.

(70) Feist, J.; Galego, J.; Garcia-Vidal, F. J. Polaritonic Chemistry with Organic Molecules. ACS Photonics 2018, 5, 205-216.

(71) Thomas, A.; Lethuillier-Karl, L.; Nagarajan, K.; Vergauwe, R. M. A.; George, J.; Chervy, T.; Shalabney, A.; Devaux, E.; Genet, C.; Moran, J.; Ebbesen, T. W. Tilting a Ground-State Reactivity Landscape by Vibrational Strong Coupling. Science 2019, 363, 615-619.

(72) Ebbesen, T. W. Hybrid Light-Matter States in a Molecular and Material Science Perspective. Acc. Chem. Res. 2016, 49, 2403-2412.

(73) Violi, I. L.; Gargiulo, J.; Von Bilderling, C.; Cortés, E.; Stefani, F. D. Light-Induced PolarizationDirected Growth of Optically Printed Gold Nanoparticles. Nano Lett. 2016, 16, 6529-6533.

(74) Martirez, J. M. P.; Carter, E. A. Prediction of a Low-Temperature N2 Dissociation Catalyst Exploiting near-IR-to-Visible Light Nanoplasmonics. Sci. Adv. 2017, 3, eaao4710. 
(75) Zhang, Y.; Nelson, T.; Tretiak, S.; Guo, H.; Schatz, G. C. Plasmonic Hot-Carrier-Mediated Tunable Photochemical Reactions. ACS Nano 2018, 12, 8415-8422.

(76) Swearer, D. F.; Zhao, H.; Zhou, L.; Zhang, C.; Robatjazi, H.; Martirez, J. M. P.; Krauter, C. M.; Yazdi, S.; McClain, M. J.; Ringe, E.; Carter, E. A.; Nordlander, P.; Halas, N. J. Heterometallic Antenna-reactor Complexes for Photocatalysis. Proc. Natl Acad. Sci. USA 2016, 113, 8916 8920.

(77) Aslam, U.; Chavez, S.; Linic, S. Controlling Energy Flow in Multimetallic Nanostructures for Plasmonic Catalysis. Nat. Nanotechnol. 2017, 12, 1000-1005.

(78) Doiron, B.; Li, Y.; Mihai, A.; Forno, S. D.; Fearn, S.; Cohen, L. F.; Alford, N. M.; Lischner, J.; Petrov, P.; Maier, S. A.; Oulton, R. F. Optimizing Hot Electron Harvesting at Planar MetalSemiconductor Interfaces with Titanium Oxynitride Thin Films. ArXiv 2018, 1807.03702v1.

(79) Doiron, B.; Li, Y.; Mihai, A.; Bower, R.; Alford, N. M.; Petrov, P. K.; Maier, S. A.; Oulton, R. F. Plasmon-Enhanced Electron Harvesting in Robust Titanium Nitride Nanostructures. J. Phys. Chem. C 2019, acs.jpcc.9b03184. 